\title{
Comprehensive Detection and Identification of Seven Animal Coronaviruses and Human Respiratory Coronavirus 229E with a Microarray Hybridization Assay
}

\author{
Qin Chen $^{\mathrm{a}}$ Jian Li ${ }^{\mathrm{b}}$ Zhirui Deng ${ }^{\mathrm{a}}$ Wei Xiong ${ }^{\mathrm{b}}$ Quan Wang ${ }^{\mathrm{c}}$ Yong-qiang Hu ${ }^{\mathrm{b}}$

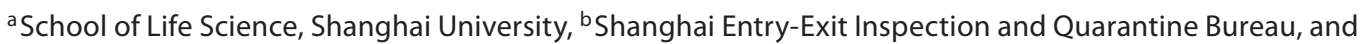 \\ 'Shanghai Veterinary Institute of CAAS, Shanghai, China
}

\section{Key Words}

Coronavirus $\cdot$ Microarray hybridization $\cdot$ Coronavirus detection

\begin{abstract}
Based on microarray hybridization, a diagnostic test for coronavirus infection was developed using eight coronavirus strains: canine coronavirus (CCoV), feline infectious peritonitis virus (FIPV), feline coronavirus (FCoV), bovine coronavirus $(\mathrm{BCoV})$, porcine respiratory coronavirus (PRCoV), turkey enteritis coronavirus (TCoV), transmissible gastroenteritis virus (TGEV), and human respiratory coronavirus (HRCoV). Up to 104 CDNA clones of eight viruses were obtained by reverse transcription PCR with different pairs of primers designed for each virus and a pair of universal primers designed for the RNA polymerase gene of coronavirus. Total RNAs extracted from virus were reverse transcribed, followed by multi-PCR amplification and labeled with Cy3-dCTP. All labeled cDNAs and prepared gene chips were subjected to specific hybridization. The results showed that extensive cross-reaction existed between CCoV, FCoV, FIPV, TGEV and PRCoV, while there was no cross-reaction between $\mathrm{BCoV}, \mathrm{TCOV}$ and $\mathrm{HRCoV}$. The ultimate specific gene chip was developed with DNA fragments reamplified from the chosen recombinant plasmids without cross-reaction between different coronaviruses.
\end{abstract}

The hybridization results showed that this gene chip could specifically identify and distinguish the eight coronaviruses and the sensitivity of the chip may be 1,000 $\times$ more sensitive than PCR, indicating that it can be used for the diagnosis of eight coronavirus infections at the same time.

Copyright $\odot 2009$ S. Karger AG, Basel

\section{Introduction}

Coronaviruses infect a number of different vertebrate species and cause economically important diseases in people, pets, livestock and poultry [1]. Especially with the explosion of SARS in 2003 in several countries, coronavirus research has been becoming more ravishing $[2,3]$. These coronaviruses are classified into three groups. One tightly clustered subgroup based on phylogenetic analysis and antigenic cross-reactivity contains canine coronavirus $(\mathrm{CCoV})$, transmissible gastroenteritis virus (TGEV) and feline coronavirus (FCoVs) $[4,5]$. In fact, all of these viruses are antigenically so similar that they may be regarded as 'host range mutants' rather than as separate species [6-9]. Coronaviruses are positive-stranded RNA viruses with exceptionally large genome sizes (up to 31 $\mathrm{kb})$. Reverse transcription-PCR (RT-PCR) is widely used for virus identification [10-12]. However, PCR cannot

\section{KARGER}

Fax +41613061234 E-Mail karger@karger.ch www.karger.com
(C) 2009 S. Karger AG, Basel

0300-5526/10/0532-0095\$26.00/0

Accessible online at:

www.karger.com/int
Qin Chen

School of Life Science, Shanghai University

Shanghai 200444 (China)

Tel. +86216613 4332, Fax +862166132177

E-Mailchenqincc@yahoo.com.cn 
distinguish several viruses at the same time from one sample and a positive amplification can be verified only by subsequent assays to elaborate sequence information. By overcoming this limitation, microarrays and biosensors have become valuable tools for viral discovery, detection, and genotyping [13-16]. Microarrays that contain several thousand different DNA sequences (probes) can theoretically identify several thousand different organisms at one time and exhibit more sensitivity and specificity than those tests which employ a single target sequence. However, the high conservation of the coronaviruses represents a design challenge and a main hindrance for the identification. It is only when coronavirus microarrays can provide information for a wide range of viral strains and have no cross-reaction between different coronaviruses that they are then usable and practical for coronavirus detection.

For this purpose, one coronavirus gene chip was developed by using $104 \mathrm{cDNA}$ clones obtained by RT-PCR technique with different primer pairs (4-15 pairs) designed for TCoV, CCoV, FCoV, FIPV, TGEV, PRCoV, $\mathrm{BCoV}$ and $\mathrm{HCoV}$, and a pair of universal primers designed for the RNA polymerase gene of coronavirus. Through two times specific hybridization, cross-reaction clones were deserted and DNA fragments reamplified from recombinant plasmids without cross-reaction between different coronaviruses were chosen to produce the ultimate specific gene chip.

\section{Materials and Methods}

\section{Virus and Cells}

Canine coronavirus (TN449 strain, ATCC No. VR-2068), feline infectious peritonitis virus (WSU 79-1146 strain, ATCC No. VR-2128) and feline coronavirus WSU79-1683 strain, ATCC No. VR-989) were propagated in a monolayer of A72 cells. Porcine respiratory coronavirus (AR310 strain, ATCC No. VR-2384) and transmissible gastroenteritis virus (Purdue strain, kept in our laboratory) were propagated in a monolayer of ST cells. Bovine coronavirus (unknown strain, ATCC No. VR-874) was propagated in a monolayer of MDBK cells. Human coronavirus (229E strain, ATCC No. VR-740) was propagated in a monolayer of MRC-5 cells. Turkey enteritis coronavirus (Minnesota strain, ATCC No. VR-911) was an original stock virus. Above eight viruses and cells were all initially obtained from American Type Culture Collection (ATCC).

\section{Primers}

Primers for RT-PCR were selected using Primer Designer (versions 1.01 and 2.01, Scientific and Educational Software, Durham, N.C., USA). Different (4-15) pairs of primers designed for each virus and a pair of universal primers designed for the RNA polymerase gene of coronavirus were used to amplify TGEV, PRCoV,
CCoV, FCoV, FIPV, BCoV, TCoV and HRCoV. Sequence analysis and alignments were done with GeneWorks version 2.5.1 (Intelligenetics, Mountain View, Calif., USA). The names and sequences of primers for all viruses used have been listed in table 1.

Construction of cDNA Clones of Eight Viruses

All 104 clones were produced by our laboratory. Escherichia coli TGI was offered by Shanghai Veterinary Institute of CAAS and pGEM-T-Easy vector bought from Promaga Co. Viral sequence data were obtained from the GenBank database. A pair of universal primers designed for the RNA polymerase gene of coronavirus and different (4-15) pairs of primers designed for each virus were used. Primers were selected to be exclusive to a given virus, as judged by pairwise BLASTN search. The total RNAs were extracted with a QIAamp Viral RNA Mini Kit (Qiagen, Germany) from TCoV stock virus and CCoV, FCoV, FIPV, TGEV, $\mathrm{PRCoV}, \mathrm{BCoV}$ and $\mathrm{HRCoV}$ purified by sucrose density gradient centrifugation were reverse transcribed (M-MLV Rtase cDNA synthesis kit, Takara Biotechnology (Dalian) Co. Ltd, China) and PCR-amplified with the above primers. The PCR products were purified and then linked with pGEM-T-Easy vector and transfected into E. coli TGI [17].

\section{Amplification and Recovery of Probe Fragments}

The PCR system was used to amplify probe fragments. The reactions were carried out in a final volume of $100 \mu$ l containing $2 \mu \mathrm{l}$ of each primer (10 pmol), $10 \times$ buffer $10 \mu \mathrm{l}$, dNTP $8 \mu \mathrm{l}$, Taq plus $2 \mu \mathrm{l}, \mathrm{dH}_{2} \mathrm{O} 71 \mu \mathrm{l}$ and $5 \mu \mathrm{l}$ plasmid template prepared by boiling lysis from different positive clones store at $-70^{\circ}$. The amplification reaction was carried out in a DNA Thermal Cycler (PerkinElmer Cetus, USA) for 30 cycles after $94^{\circ}$ for 5 min with denaturation at $94^{\circ}$ for $45 \mathrm{~s}$, annealing at $52^{\circ}$ for $45 \mathrm{~s}$ and polymerization at $72^{\circ}$ for $90 \mathrm{~s}$. A final extension at $72^{\circ}$ for $10 \mathrm{~min}$ was carried out before holding the samples at $4^{\circ}$. The amplification reaction of universal primers designed for the RNA polymerase gene was 5 cycles after $94^{\circ}$ for 5 min with denaturation at $94^{\circ}$ for $45 \mathrm{~s}$, annealing at $40^{\circ}$ for $45 \mathrm{~s}$ and polymerization at $72^{\circ}$ for $60 \mathrm{~s}$, then 30 cycles with denaturation at $94^{\circ}$ for $45 \mathrm{~s}$, annealing at $50^{\circ}$ for $60 \mathrm{~s}$, polymerization at $72^{\circ}$ for $60 \mathrm{~s}$ and a final extension at $72^{\circ}$ for $10 \mathrm{~min}$. The amplified product was recovered using PCR Kleen $^{\mathrm{TM}}$ spin columns (Bio-Rad Laboratories, Inc., USA). The PCR product was cleaned with a Qiagen QIAquick PCR purification kit. These fragments were designed to have similar annealing stabilities.

\section{Preparation of Probes and Spotting on Chips}

The concentrations of the above probe fragments were determined by $\mathrm{OD}_{260}$ and $\mathrm{OD}_{280}$, then dried and suspended in 300 $\mathrm{ng} / \mu \mathrm{l}$ with $50 \%$ DMSO. Spotting was completed using a Bio-Rad printer on slides coated with amino saline. The slides were UV cross-linked at $60 \mathrm{~mJ}$ for $25 \mathrm{~min}$ and baked for $2 \mathrm{~h}$ at $80^{\circ}$, bathed for $3 \mathrm{~min}$, immediately put into cold absolute ethanol or a refrigerator for $2 \mathrm{~min}$, centrifuged $8 \mathrm{~min}$ at 1,000 rpm, dried, a prehybridization solution was added on the slide, prehybridized at $42^{\circ}$ for $1 \mathrm{~h}$, then rinsed twice with distilled water, centrifuged at 1,000 $\mathrm{rpm}$, and stored in a dry dust-free environment. The same virus probes were a designed neighborhood and some QC (quality control, $10 \mu \mathrm{M}$ HEX), BC (blank control, 50\% DMSO), NC (negative control, 127 (SARS)) and EC (PCR product of HLA) were arrayed (see table 2). 
Table 1. Names and sequence of primers for all viruses

\begin{tabular}{|c|c|c|c|}
\hline $\begin{array}{l}\text { Primer } \\
\text { sets }\end{array}$ & Sequence of primer & $\begin{array}{l}\text { Primer } \\
\text { sets }\end{array}$ & Sequence of primer \\
\hline FCoV1 & $\begin{array}{l}\text { Fw primer } 5^{\prime} \text {-TGATTGTGCTCGTAACTTGC-3' } \\
\text { Rw primer } 5^{\prime} \text {-CTGGTGAAGGTGTTATAGTC-3' }\end{array}$ & FCoV2 & $\begin{array}{l}\text { Fw primer } 5^{\prime} \text {-TAACACCTTCACCAGCAACC-3' } \\
\text { Rw primer 5'-GAGCAAAGACATTAGTGGCG-3' }\end{array}$ \\
\hline FCoV3 & $\begin{array}{l}\text { Fw primer 5'-GGAGGTTACATACCTGATGG-3' } \\
\text { Rw primer } 5^{\prime} \text {-AGTACATAACAGTACCGTGG-3' }\end{array}$ & FCoV4 & $\begin{array}{l}\text { Fw primer 5'-TGCTATTAGTAAGTGGGGCC-3' } \\
\text { Rw primer 5' } 5^{\prime} \text {-ATAACCGCTACGCTTCATAC-3' }\end{array}$ \\
\hline FCoV5 & $\begin{array}{l}\text { Fw primer 5'-CATTACACTACCAATGCAGG-3' } \\
\text { Rw primer 5' } 5^{\prime} \text {-ATACCAACACCAGTTCTACC-3' }\end{array}$ & FCoV6 & 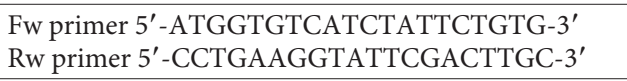 \\
\hline FCoV7 & $\begin{array}{l}\text { Fw primer 5' -CACCACACCAGTATCAATAG-3' } \\
\text { Rw primer 5' } 5^{\prime} \text {-CTACACCAGGTAACACCATG-3' }\end{array}$ & FCoV8 & $\begin{array}{l}\text { Fw primer 5'-TCTCTTGCAGGTGGTATAAC-3' } \\
\text { Rw primer 5' } 5^{\prime} \text {-GCTTGTCTGGTTAGAGTCTG-3' }\end{array}$ \\
\hline FCoV9 & $\begin{array}{l}\text { Fw primer 5'-GGTTAGGGCTAGTAGACAAC-3' } \\
\text { Rw primer 5' } 5^{\prime} \text {-TGACAAACAACACATCGCAC-3' }\end{array}$ & FCoV10 & $\begin{array}{l}\text { Fw primer 5'-GACTGTACCTGAATTGACAC-3' } \\
\text { Rw primer 5' -CTCTAGCAGAAACAACTGTC-3' }\end{array}$ \\
\hline FCoV11 & $\begin{array}{l}\text { Fw primer 5' -CCATAACTATGACGTTCCCC-3' } \\
\text { Rw primer 5' -TGCCAAATAAGATCACCTCC-3' }\end{array}$ & FCoV12 & 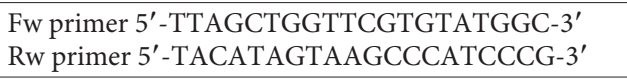 \\
\hline FCoV13 & $\begin{array}{l}\text { Fw primer 5' -CTCAACAGAAGCACGTACTG-3' } \\
\text { Rw primer 5' } 5^{\prime} \text {-AGACACCGTCAATCTTAGCC-3' }\end{array}$ & FCoV14 & $\begin{array}{l}\text { Fw primer 5' } \text { 5 AACATCACTTGGCACTCGTG-3' }^{\prime} \text { Rw primer 5' -GCTGAACTACTTCTAGCACC-3' } \\
\text { Rw }\end{array}$ \\
\hline FCoV15 & $\begin{array}{l}\text { Fw primer 5' } 5^{\prime} \text { AGTGATCTCGTTGCCAATGG-3' } \\
\text { Rw primer } 5^{\prime} \text {-CATGCGTTTAGTTCGTTACC-3' }\end{array}$ & TGEV1 & $\begin{array}{l}\text { Fw primer 5' -GTGGTTTTGGTCGTAATGCC-3' } \\
\text { Rw primer 5' } 5^{\prime} \text {-TGGTGGTAGTAGGTGGTGAG-3' }\end{array}$ \\
\hline TGEV2 & $\begin{array}{l}\text { Fw primer 5'-AATTGGGGTAGTGAGTGCAG-3' } \\
\text { Rw primer 5' } 5^{\prime} \text {-CCAACGTGGAGCTATTAGTT-3' }\end{array}$ & TGEV3 & $\begin{array}{l}\text { Fw primer 5' }{ }^{\prime} \text {-TACAATCAGGTAAGGGTGCC-3' } \\
\text { Rw primer 5' } 5^{\prime} \text {-ACGTCACTATCACCAGTGGT-3' }\end{array}$ \\
\hline TGEV4 & $\begin{array}{l}\text { Fw primer 5'-ACCACTGGTGATAGTGACGT-3' } \\
\text { Rw primer 5' -GGTCAGAACGAATACAGTAC-3' }\end{array}$ & TGEV5 & $\begin{array}{l}\text { Fw primer 5' }{ }^{\prime} \text {-ACAACATAGTGGGTGTACCG-3' } \\
\text { Rw primer 5' } 5^{\prime} \text {-GCCACGAGTCCTATCATTTG-3' }\end{array}$ \\
\hline TGEV6 & $\begin{array}{l}\text { Fw primer 5' -GTGTTCCATCTGTGTCTAGC-3' } \\
\text { Rw primer 5' -CAACCTGTGTGTCATCAAAC-3' }\end{array}$ & TGEV7 & $\begin{array}{l}\text { Fw primer 5'-GGCGATCTTATTTGGCATCT-3' } \\
\text { Rw primer 5' -CCACCAAGACTTAGTCCTTC-3' }\end{array}$ \\
\hline TGEV8 & $\begin{array}{l}\text { Fw primer 5' -GGGAATTTGTACGCTGAAGG-3' } \\
\text { Rw primer 5' } 5^{\prime} \text {-TCTTCCGACCACGGGAATTG-3' }\end{array}$ & TGEV9 & $\begin{array}{l}\text { Fw primer 5'-GACACTTTTAGGACCTATGC-3' } \\
\text { Rw primer 5' } 5^{\prime} \text {-TAAGCCACGTATTGCTATGC-3' }\end{array}$ \\
\hline TGEV10 & $\begin{array}{l}\text { Fw primer 5'-TTTTCACAGGAGCCCGTAGT-3' } \\
\text { Rw primer 5' } 5^{\prime} \text {-AGGCATCGTAAGCATGTTGC-3' }\end{array}$ & BCoV1 & $\begin{array}{l}\text { Fw primer 5'-CCCCCGTACTGTTATTTTCG-3' } \\
\text { Rw primer 5' } 5^{\prime} \text {-TGGTCTAAGCATCATGCAGC-3' }\end{array}$ \\
\hline $\mathrm{BCoV} 2$ & $\begin{array}{l}\text { Fw primer 5'-GGCTTTTGCTGTTATAGGAG-3' } \\
\text { Rw primer 5' } 5^{\prime} \text {-CTTGGTATTTTTGACCTTAGC-3' }\end{array}$ & BCoV3 & $\begin{array}{l}\text { Fw primer 5'-GCGTAGAACTATGGCATTGG-3' } \\
\text { Rw primer 5' } 5^{\prime} \text {-ATACGTCGGTAAACATCTGC-3' }\end{array}$ \\
\hline$\overline{\mathrm{BCoV} 4}$ & $\begin{array}{l}\text { Fw primer 5' } 5^{\prime} \text {-TTAATGATAAGTCGGTGCCC-3' } \\
\text { Rw primer } 5^{\prime} \text {-CATCCAATTTACACGGACAG-3' }\end{array}$ & BCoV5 & $\begin{array}{l}\text { Fw primer R 5'-GATGGGTCTTTGTGTGTAGG-3' } \\
\text { Rw primer 5' } \text { 5'ACACCTATCCCCTTGTAAAC-3' }^{\prime}\end{array}$ \\
\hline BCoV6 & $\begin{array}{l}\text { Fw primer 5'-TGTTTACAAGGGGATAGGTG-3' } \\
\text { Rw primer 5' } 5^{\prime} \text {-AAGAGTTAGCATGAAAGGCC-3' }\end{array}$ & BCoV7 & $\begin{array}{l}\text { Fw primer 5'-GCCTTTCATGCTAACTCTTC-3' } \\
\text { Rw primer 5' } 5^{\prime} \text {-TACAAACCACCTACAGGTTC-3' }\end{array}$ \\
\hline$\overline{\mathrm{BCoV} 8}$ & $\begin{array}{l}\text { Fw primer 5' } 5^{\prime} \text {-ACCTGTAGGTGGTTTGTATG-3' } \\
\text { Rw primer 5'-CCGACATCAGATAACTTTAC-3' }\end{array}$ & BCoV9 & $\begin{array}{l}\text { Fw primer 5'-TAATTGTACTGGAGGTGCCG-3' } \\
\text { Rw primer 5' } \text { 5.TCTATCTGAGCTTGCGCTTC-3' }^{\prime}\end{array}$ \\
\hline $\mathrm{BCoV} 10$ & $\begin{array}{l}\text { Fw primer 5'-GCGTCTTACCGCTCTTAATG-3' } \\
\text { Rw primer 5' } 5^{\prime} \text {-ACTACCAGTGAACATCCAAG-3' }\end{array}$ & BCoV11 & $\begin{array}{l}\text { Fw primer 5'-ATGAATAGGTTACAGGAGGC-3' } \\
\text { Rw primer 5' } 5^{\prime} \text {-CCACTAAACAGCAGGCATTG-3' }\end{array}$ \\
\hline $\mathrm{BCoV} 12$ & $\begin{array}{l}\text { Fw primer } 5^{\prime} \text {-ACTGCCATCAACCCAAAAGG-3' } \\
\text { Rw primer 5' } 5^{\prime} \text {-TCTGTACCAGTACCCCTTAG-3' }\end{array}$ & $\mathrm{BCoV} 13$ & $\begin{array}{l}\text { Fw primer 5'-TACTATCTTGGAACAGGACC-3' } \\
\text { Rw primer 5' } 5^{\prime} \text {-GCTTAGTTACTTGCTGTGGC-3' }\end{array}$ \\
\hline BCoV14 & $\begin{array}{l}\text { Fw primer 5'-CCCCAATAAACAATGCACTG-3' } \\
\text { Rw primer 5' -GTGGTTTTGGACTCATATTC-3' }\end{array}$ & BCoV15 & $\begin{array}{l}\text { Fw primer 5'-AATATAAGTGTTGCAGCGCC-3' } \\
\text { Rw primer 5' } 5^{\prime} \text {-TTAACATGCTGGCTCTTCCC-3' }\end{array}$ \\
\hline $\mathrm{CCoV} 1$ & $\begin{array}{l}\text { Fw primer 5' } \text { 'ACGTGGTCGTTCCAATTCTC-3' }^{\prime} \text { ACG } \\
\text { Rw primer 5'-GTCCCTTGATTGGTTCACTTC-3' }\end{array}$ & $\mathrm{CCoV} 2$ & $\begin{array}{l}\text { Fw primer 5'-GCTGCACTCAAAAAGTTAGG-3' } \\
\text { Rw primer 5'-TACAACCTCTTGCTCTACCC-3' }\end{array}$ \\
\hline $\mathrm{CCoV} 3$ & $\begin{array}{l}\text { Fw primer 5' -AGGAACATTACCACCCAGTG-3' } \\
\text { Rw primer 5' -AACGAGACCTACTTCACTTG-3' }\end{array}$ & $\mathrm{CCoV} 4$ & $\begin{array}{l}\text { Fw primer 5'-GGTAGAACTGGTGTTGGTAT-3' } \\
\text { Rw primer 5' -CCACGAGTCCTCTCATTTGT-3' }\end{array}$ \\
\hline$\overline{\mathrm{CCoV} 5}$ & $\begin{array}{l}\text { Fw primer 5'-TGATTGTGCTCGTAACTTGC-3' } \\
\text { Rw primer 5' } \text { 5.TCATCATTCCACTCAAGCCC-3' }^{\prime}\end{array}$ & CCoV6 & $\begin{array}{l}\text { Fw primer 5'-CTACATGGCAACACAGTGCT-3' } \\
\text { Rw primer 5' -GCCGAATGGAATTTCACCAT-3' }\end{array}$ \\
\hline$\overline{\mathrm{CCoV} 7}$ & $\begin{array}{l}\text { Fw primer } 5^{\prime} \text {-AGAGCTGCACCGTTTATGAG-3' } \\
\text { Rw primer } 5^{\prime} \text {-TATGACAGTCAATGCCCGAG-3' }\end{array}$ & FIPV1 & $\begin{array}{l}\text { Fw primer 5'-GGTTAGGGCTAGTAGACAAC-3' } \\
\text { Rw primer 5' } 5^{\prime} \text {-AACAACACATCACACCCTTC-3' }\end{array}$ \\
\hline FIPV2 & $\begin{array}{l}\text { Fw primer 5'-TGTCAACGCGACTGTAATTG-3' } \\
\text { Rw primer 5' } 5^{\prime} \text {-CAACAACTTCCTAAACAACC-3' }\end{array}$ & FIPV3 & $\begin{array}{l}\text { Fw primer 5'-TTATGGTGAACGCTACTGTG-3' } \\
\text { Rw primer 5' } 5^{\prime} \text {-CATCATCCAAAGTGCAAACG-3' }\end{array}$ \\
\hline FIPV4 & $\begin{array}{l}\text { Fw primer 5'-GAAGA(G)ACCAAATCATGGTGG-3' } \\
\text { Rw primer 5' } 5^{\prime} \text {-AAGGTTCATCTCCCCAGTTG-3' }\end{array}$ & FIPV5 & $\begin{array}{l}\text { Fw primer 5'-TGATGGA(T)GTCTTCTGGGTTG-3' } \\
\text { Rw primer 5' } 5^{\prime} \text {-TTCCAGGTGTGTTTGTTGGC-3' }\end{array}$ \\
\hline FIPV6 & $\begin{array}{l}\text { Fw primer 5'-AGTGATCTCGTTGCCAATGG-3' } \\
\text { Rw primer 5' } 5^{\prime} \text {-TTACAAGTACAGCATGGACG-3' }\end{array}$ & FIPV7 & $\begin{array}{l}\text { Fw primer 5' } 5^{\prime} \text {-ACTGCGAGTGATCTTTCTAG-3' } \\
\text { Rw primer 5'-TTTTGTTTTGGCACAGCAC-3' }\end{array}$ \\
\hline
\end{tabular}


Table 1 (continued)

\begin{tabular}{|c|c|c|c|}
\hline $\begin{array}{l}\text { Primer } \\
\text { sets }\end{array}$ & Sequence of primer & $\begin{array}{l}\text { Primer } \\
\text { sets }\end{array}$ & Sequence of primer \\
\hline FIPV8 & $\begin{array}{l}\text { Fw primer 5' } \text {-AGATTAGTTGGTGCTGTGCC-3' }^{\prime} \\
\text { Rw primer 5' } 5^{\prime} \text {-ATAGGGTTGCTTGTACCTCC-3' }\end{array}$ & FIPV9 & $\begin{array}{l}\text { Fw primer 5' -TGACAGGGATTTTCAACACC-3' } \\
\text { Rw primer R 5'-ACAATCACTAGATCCAGACG-3' }\end{array}$ \\
\hline FIPV10 & $\begin{array}{l}\text { Fw primer 5'-ACATTACAACACACCAGGTC-3' } \\
\text { Rw primer 5'-GTTGAAAATCCCTGTCATGG-3' }\end{array}$ & FIPV11 & $\begin{array}{l}\text { Fw primer 5'-GGTTGAGATGATTGATGAGG-3' } \\
\text { Rw primer 5'-CCTGGTGTGTTGTAATGTAG-3' }\end{array}$ \\
\hline PRCoV1 & $\begin{array}{l}\text { Fw primer 5'-GTGGTTTTGGTT(C)G(A)TAATGCC-3' } \\
\text { Rw primer 5'-CCTTCTTCAAAGCTAGGGAC-3' }\end{array}$ & PRCoV2 & $\begin{array}{l}\text { Fw primer 5'-CCGTGGATGT(C)TGTACTTGAC-3' } \\
\text { Rw primer 5' -GGTCTTGTTGTGTATGATGC-3' }\end{array}$ \\
\hline PRCoV3 & $\begin{array}{l}\text { Fw primer 5'-GCATCATACACAACAAGACC-3' } \\
\text { Rw primer 5'-TTCTCTTAAACGGTGCAGCT-3' }\end{array}$ & PRCoV4 & $\begin{array}{l}\text { Fw primer 5'-GATTCGCCTACTTAGCATAC-3' } \\
\text { Rw primer 5'-CCTGAGAAAAGGCTGCATTG-3' }\end{array}$ \\
\hline TCoV1 & $\begin{array}{l}\text { Fw primer 5'-CAGATGCTCAGGTTGATAGG-3' } \\
\text { Rw primer 5' } \text { 5.TGATGCCACTTCCAGTCTTG-3' }^{\prime}\end{array}$ & TCoV2 & $\begin{array}{l}\text { Fw primer 5'-ACCCTTTCAACCAGCATTGC-3' } \\
\text { Rw primer 5' } 5^{\prime} \text {-CCACACTTACTCATTAGCGG-3' }\end{array}$ \\
\hline $\mathrm{TCoV} 3$ & $\begin{array}{l}\text { Fw primer 5' } 5^{\prime} \text {-TCAGTGGCTTGCTAAGTGTG-3' } \\
\text { Rw primer 5'-CCTTCGTAGTAGACTTTTCC-3' }\end{array}$ & TCoV4 & $\begin{array}{l}\text { Fw primer 5'-AGTGTAGCAACAGGAGGAAG-3' } \\
\text { Rw primer 5'-CAAAATCGCTCGCCTACTAC-3' }\end{array}$ \\
\hline TCoV5 & $\begin{array}{l}\text { Fw primer 5' -GTAGTAGGCGAGCGATTTTG-3' } \\
\text { Rw primer 5' } \text { 5AACTTTAGGTGGCTTTGGTC-3' }^{\prime}\end{array}$ & TCoV6 & $\begin{array}{l}\text { Fw primer 5'-ACCAAAGCCACCTAAAGTTG-3' } \\
\text { Rw primer 5'-GAGGAATAAAGTCCCAACGG-3' }\end{array}$ \\
\hline TCoV7 & $\begin{array}{l}\text { Fw primer 5' } \text { 5TCCTCTGAATCGTGGTAGG-3' }^{\prime} \text {-TCCACGCCATCCGTAATAC-3' } \\
\text { Rw primer 5' }\end{array}$ & TCoV8 & $\begin{array}{l}\text { Fw primer 5' } \text { 5 ATGGGCGTGTTACAGCAATG-3' }^{\prime} \\
\text { Rw prime 5' -CTACCTCATCATCCTGCTTC-3' }\end{array}$ \\
\hline TCoV9 & $\begin{array}{l}\text { Fw primer 5' -GAAGCAGGATGATGAGGTAG-3' } \\
\text { Rw primer 5' -ATGCCTATCTGCCTTAACTC-3' }\end{array}$ & HRCoV1 & $\begin{array}{l}\text { Fw primer 5'-CGGGATCCGAAGGTGTCGTCTGGGTTGC } \\
\text { Rw primer 5' } \text {-CCGCTCGAGCTGCACTTCCAAAGTTGTG }\end{array}$ \\
\hline HRCoV2 & $\begin{array}{l}\text { Fw primer 5' } \text {-CGGGATCCATGTTTGTTTTGCTTGTTG } \\
\text { Rw primer 5'-CCGCTCGAGGAAGCTAACGCAACAGTAC }\end{array}$ & HRCoV3 & $\begin{array}{l}\text { Fw primer 5'-CGGGATCCACTGGCGTCCCACAACCTG } \\
\text { Rw prime 5'-CCGCTCGAGGTGAGCATCTCACTAACATC }\end{array}$ \\
\hline HRCoV4 & $\begin{array}{l}\text { Fw primer 5' }- \text {-CGGGATCCAGCAAACTTGTTACTTCTGG } \\
\text { Rw primer 5'-CCGCTCGAGGTCTGGAAGCACGAACTTC }\end{array}$ & HRCoV5 & $\begin{array}{l}\text { Fw primer 5'-CGGGATCCTGTGGAAATGGCACTCACA } \\
\text { Rw primer 5' }{ }^{\prime} \text {-CGCTCGAGCAACGTCGTAATAAGGAAG }\end{array}$ \\
\hline HRCoV6 & $\begin{array}{l}\text { Fw primer 5'-CGGGATCCCAGAACAATGAAATAATGCC } \\
\text { Rw primer 5'-CCGCTCGAGGTCCGGTCACATGTACAGC }\end{array}$ & HRCoV7 & $\begin{array}{l}\text { Fw primer } 5^{\prime} \text {-CGGGATCCCACAAAAGGGTGATGCTGC } \\
\text { Rw primer } 5 \text {-CCGCTCGAGCATCACCAGAAGTTGTACCACC }\end{array}$ \\
\hline HRCoV8 & $\begin{array}{l}\text { Fw primer } 5^{\prime} \text {-CGGGATCCCTATGATGATTCTTTCTGATG } \\
\text { Rw primer 5'-CCGCTCGAGTGTGTTACACACATATGGTG }\end{array}$ & HRCoV9 & $\begin{array}{l}\text { Fw primer 5'-CGGGATCCGACTACAAGCTTGCTAATG } \\
\text { Rw primer 5'-CCGCTCGAGGCACAGAGCGAATCAACAGC }\end{array}$ \\
\hline HRCoV10 & $\begin{array}{l}\text { Fw primer 5' -CGGGATCCGACATTGTTGTCGTGGATG } \\
\text { Rw primer 5' -CCGCTCGAGCTTTGCTCTAGTAATGGCAAC }\end{array}$ & HRCoV11 & $\begin{array}{l}\text { Fw primer 5'-CGGGATCCGATGTGGAAGGTGCACATG } \\
\text { Rw primer 5' -CCGCTCGAGCATATCCACATTACAATTCC }\end{array}$ \\
\hline HRCoV12 & $\begin{array}{l}\text { Fw primer 5'-CGGGATCCGGTGCTGATGGTGAATTACC } \\
\text { Rw primer 5' } 5^{\prime} \text {-CCGCTCGAGTCACCATACACAACGTGCTC }\end{array}$ & HRCoV13 & $\begin{array}{l}\text { Fw primer 5'-CGGGATCCATCCTCAGTTGCAGAGTGC } \\
\text { Rw primer 5' -CCGCTCGAGCCATCTATAAAAGGTCCTTG }\end{array}$ \\
\hline HRCoV14 & $\begin{array}{l}\text { Fw primer 5'-CGGGATCCATGTCAAATGACAATTGTAC } \\
\text { Rw primer 5'-CCGCTCGAGGAAAAAATGAAGCAATCTTTC }\end{array}$ & HRCoV15 & $\begin{array}{l}\text { Fw primer 5' -CGGGATCCGATCTTAATGCTAATATGTC } \\
\text { Rw primer 5' -CCGCTCGAGGCCAAACTTAGCATAATGCC }\end{array}$ \\
\hline $\mathrm{UP}$ & $\begin{array}{l}\text { FW primer } 5^{\prime} \text {-ACTCA(A/G)(A/T)T(A/G)AAT(T/C)TNAAATA } \\
\text { Rw primer } 5^{\prime} \text {-TCACA }(\mathrm{C} / \mathrm{T}) \mathrm{TT}(\mathrm{A} / \mathrm{T}) \mathrm{GGATA}(\mathrm{G} / \mathrm{A}) \mathrm{TCCCA}\end{array}$ & $(\mathrm{T} / \mathrm{C}$ & \\
\hline
\end{tabular}

Table 2. Array arrangement of primary coronaviruses chip

\begin{tabular}{|c|c|c|c|c|c|c|c|c|c|c|c|c|c|c|c|c|}
\hline & 1 & 2 & 3 & 4 & 5 & 6 & 7 & 8 & 9 & 10 & 11 & 12 & 13 & 14 & 15 & 16 \\
\hline A & QC & QC & $\mathrm{BCoV} 1$ & BCoV1 & UP1 & UP1 & QC & QC & QC & QC & FCoV1 & FCoV1 & HRCoV1 & HRCoV1 & QC & QC \\
\hline B & $\mathrm{NC1}$ & $\mathrm{NC} 1$ & BCoV2 & BCoV2 & UP2 & UP2 & FIPV1 & FIPV1 & PRCoV1 & PRCoV1 & FCoV2 & FCoV2 & HRCoV2 & HRCoV2 & $\mathrm{NC} 2$ & $\mathrm{NC} 2$ \\
\hline $\mathrm{C}$ & TGEV1 & TGEV1 & $\mathrm{BCoV} 3$ & $\mathrm{BCoV} 3$ & UP3 & UP3 & FIPV2 & FIPV2 & PRCoV2 & PRCoV2 & FCoV3 & FCoV3 & HRCoV3 & HRCoV3 & $\mathrm{BC}$ & $\mathrm{BC}$ \\
\hline D & TGEV2 & TGEV2 & BCoV4 & BCoV4 & UP4 & UP4 & FIPV3 & FIPV3 & PRCoV3 & PRCoV3 & FCoV4 & FCoV4 & HRCoV4 & HRCoV4 & TCoV1 & TCoV1 \\
\hline E & TGEV3 & TGEV3 & BCoV5 & BCoV5 & UP5 & UP5 & FIPV4 & FIPV4 & PRCoV4 & PRCoV4 & FCoV5 & FCoV5 & HRCoV5 & HRCoV5 & TCoV2 & TCoV2 \\
\hline $\mathrm{F}$ & TGEV4 & TGEV4 & BCoV6 & BCoV6 & UP6 & UP6 & FIPV5 & FIPV5 & CCoV1 & CCoV1 & FCoV6 & FCoV6 & HRCoV6 & HRCoV6 & TCoV3 & TCoV3 \\
\hline G & TGEV5 & TGEV5 & BCoV7 & BCoV7 & UP7 & UP7 & FIPV6 & FIPV6 & CCoV2 & $\mathrm{CCoV} 2$ & FCoV7 & FCoV7 & HRCoV7 & HRCoV7 & TCoV4 & TCoV4 \\
\hline $\mathrm{H}$ & EC & $\mathrm{EC}$ & BCoV8 & BCoV8 & UP8 & UP8 & $\mathrm{BC}$ & $\mathrm{BC}$ & $\mathrm{CCoV} 3$ & $\mathrm{CCoV} 3$ & FCoV8 & FCoV8 & HRCoV8 & HRCoV8 & EC & EC \\
\hline I & TGEV6 & TGEV6 & BCoV9 & BCoV9 & UP9 & UP9 & FIPV7 & FIPV7 & $\mathrm{CCoV} 4$ & CCoV4 & FCoV9 & FCoV9 & HRCoV9 & HRCoV9 & TCoV5 & TCoV5 \\
\hline $\mathrm{J}$ & TGEV7 & TGEV7 & BCoV10 & BCoV10 & UP10 & UP10 & FIPV8 & FIPV8 & CCoV5 & CCoV5 & FCoV10 & FCoV10 & HRCoV10 & HRCoV10 & TCoV6 & TCoV6 \\
\hline $\mathrm{K}$ & TGEV8 & TGEV8 & BCoV11 & BCoV11 & UP11 & UP11 & FIPV9 & FIPV9 & CCoV6 & CCoV6 & FCoV11 & FCoV11 & HRCoV11 & HRCoV11 & TCoV7 & TCoV7 \\
\hline $\mathrm{L}$ & TGEV9 & TGEV9 & BCoV12 & BCoV12 & UP12 & UP12 & FIPV10 & FIPV10 & CCoV7 & CCoV7 & FCoV12 & FCoV12 & HRCoV12 & HRCoV12 & TCoV8 & TCoV8 \\
\hline M & TGEV10 & TGEV10 & BCoV13 & BCoV13 & UP13 & UP13 & FIPV11 & FIPV11 & CCoV1 & CCoV1 & FCoV13 & FCoV13 & HRCoV13 & HRCoV13 & TCoV9 & TCoV9 \\
\hline $\mathrm{N}$ & NC3 & NC3 & BCoV14 & BCoV14 & UP14 & UP14 & UP16 & UP16 & CCoV2 & CCoV2 & FCoV14 & FCoV14 & HRCoV14 & HRCoV14 & NC4 & NC4 \\
\hline $\mathrm{O}$ & QC & QC & BCoV15 & BCoV15 & UP15 & UP15 & QC & QC & QC & QC & FCoV15 & FCoV15 & HRCoV15 & HRCoV15 & QC & QC \\
\hline
\end{tabular}


Table 3. Hybridization and amplification of multi-PCR of different coronavirus

\begin{tabular}{cll}
\hline Group & Primer sets & Cross-reaction gene clones of other viruses \\
\hline 1 & BCoV1, BCoV2, BCoV3, BCoV4, BCoV5 & NO \\
2 & BCoV6, BCoV7, BCoV8, BCoV9, BCoV10 & NO \\
3 & BCoV11, BCoV12, BCoV13, BCoV14, BCoV15 & NO \\
4 & TCoV1, TCoV2, TCoV3, TCoV4, TCoV5 & NO \\
5 & TCoV6, TCoV7, TCoV8, TCoV9 & NO \\
6 & CCoV1, CCoV2, CCoV3, CCoV4, CCoV5 & TGEV3, TGEV4, TGEV5, TGEV6, TGEV8, FIPV9, FCoV2, \\
& & FCoV4, FCoV9, FCoV10, FCoV11 \\
7 & CCoV6, CCoV7 & FIPV3, FIPV4, TGEV4, TGEV5, TGEV7, TGEV8, TGEV9, \\
& & TGEV10, FCoV2, FCoV3, FCoV9 \\
8 & FIPV1, FIPV2, FIPV3, FIPV4, FIPV5 & CCoV1, CCoV2, TGEV7, TGEV8, FCoV7, FCoV9, FCoV10, \\
9 & FIPV6, FIPV7, FIPV8, FIPV9, FIPV10, FIPV11 & FCoV11, FCoV12 \\
10 & FCoV1, FCoV2, FCoV3, FCoV4, FCoV5 & CCoV6 \\
11 & FCoV6, FCoV7, FCoV8, FCoV9, FCoV10 & TGEV7, TGEV10, FIPV1, FIPV3, FIPV4 \\
12 & FCoV11, FCoV12, FCoV13, FCoV14, FCoV15 & TGEV6, TGEV8, TGEV9, TGEV10, CCoV1, CCoV2, CCoV7, \\
& & FIPV4, FIPV5, FIPV6, FIPV7, FIPV8 \\
13 & TGEV1, TGEV2, TGEV3, TGEV4, TGEV5 & CCoV3, CCoV4, FIPV3, FIPV4 \\
14 & TGEV6, TGEV7, TGEV8, TGEV9, TGEV10 & FIPV4, CCoV1, CCoV2, CCoV7, FCoV10 \\
15 & HRCoV1, HRCoV6, HRCoV8, HRCoV13, HRCoV15 & UP2, UP7 \\
16 & HRCoV2, HRCoV3, HRCoV4, HRCoV5, HRCoV14 & NO \\
17 & HRCoV7, HRCoV9, HRCoV10, HRCoV11, HRCoV12 & NO \\
18 & PRCoV1, PRCoV2, PRCoV3, PRCoV4 & TGEV9, CCoV7 \\
& &
\end{tabular}

$\mathrm{NO}=$ No cross-reaction was observed with gene clones from other viruses.

\section{Samples Processing and Hybridizing}

Viral RNAs were extracted from the cryolysate of cell cultures infected with the examined coronavirus strains using a QIAamp Viral RNA Mini Kit (Qiagen, Germany) and reverse transcribed with M-MLV Rtase cDNA synthesis kit (Takara Biotechnology (Dalian) Co. Ltd., China), with oligo-dT as primer, then each cDNA was multi-PCR amplified with different primer sets (see table 3; each virus cDNA with different primer sets, such as $\mathrm{CCoV}$ with $\mathrm{CCoV} 1, \mathrm{CCoV} 2, \mathrm{CCoV} 3, \mathrm{CCoV} 4, \mathrm{CCoV} 5$ primer mixture, and $\mathrm{CCoV} 6, \mathrm{CCoV} 7$ primer mixture to amplify separately) and labeled with Cy3-dCTP (PerkinElmer). The labeled fluorescent DNAs were denatured at $96^{\circ}$ for $5 \mathrm{~min}$, then placed on a slide and put into a hybridization chamber oven at $42^{\circ}$ for $2-3 \mathrm{~h}$. Different multi-PCR samples with Cy3-dCTP were hybridized with the chip. After washing, the slides were scanned on a GenePix 4000B array scanner (Packard Biochip Technologies, Mass., USA) $(\lambda \operatorname{ex} 543 \mathrm{~nm} / \lambda \operatorname{ex} 570 \mathrm{~nm})$ at $10 \mu \mathrm{m}$ resolution and analyzed with GenePix Pro array analysis software.

Sensitivity and Application of the Coronavirus Chip

The 10 -fold serial $\mathrm{BCoV}$ cDNA dilution was detected by multi-PCR and the coronavirus gene chip using the same primer set. The results were shown by electrophoresis in a $1 \%$ agarose gel for $1 \mathrm{~h}$ at $90 \mathrm{~V}$, visualized by ethidium bromide and a slide reader. Muscle, heart, liver, spleen, lung and kidney were gathered from the pigs infected with live TGEV, PRCoV, canines infected with live $\mathrm{CCoV}$, felines infected with live FCoV, FIPV, and cattle infected with live $\mathrm{BCoV}$, which were detected with RTPCR, cell culture and microassay, while 10 canine hearts (7 positive and 3 negative to $\mathrm{CCoV}$ analyzed by PCR), 5 feline lungs ( 3 positive and 2 negative to FIPV analyzed by PCR) and 6 pig lungs (5 positive and 1 negative to $\mathrm{PRCoV}$ analyzed by PCR) were collected from clinical animals and detected with microassay [12, $13,15]$.

\section{Results}

Hybridizing to Different Multi-PCR Samples with the Primary Coronavirus Chip

Different multi-PCR samples with Cy3-dCTP were hybridized with the chip. The slides were scanned by a ScanArray 4000c laser scanning system (Packard Biochip Technologies) with a laser for Cy3 dye ( $\lambda$ ex543 nm/ $\lambda \operatorname{ex} 570 \mathrm{~nm})$ at $10 \mu \mathrm{m}$ resolution. Different intense signals appeared. From the hybridization, extensive cross-reactions between $\mathrm{CCoV}, \mathrm{FCoV}$, FIPV, TGEV and PRCoV were found, while there were none between $\mathrm{BCoV}, \mathrm{TCoV}$ and $\mathrm{HRCoV}$. The results are shown in table 3 and figure $1 \mathrm{a}-\mathrm{c}$ as samples. 

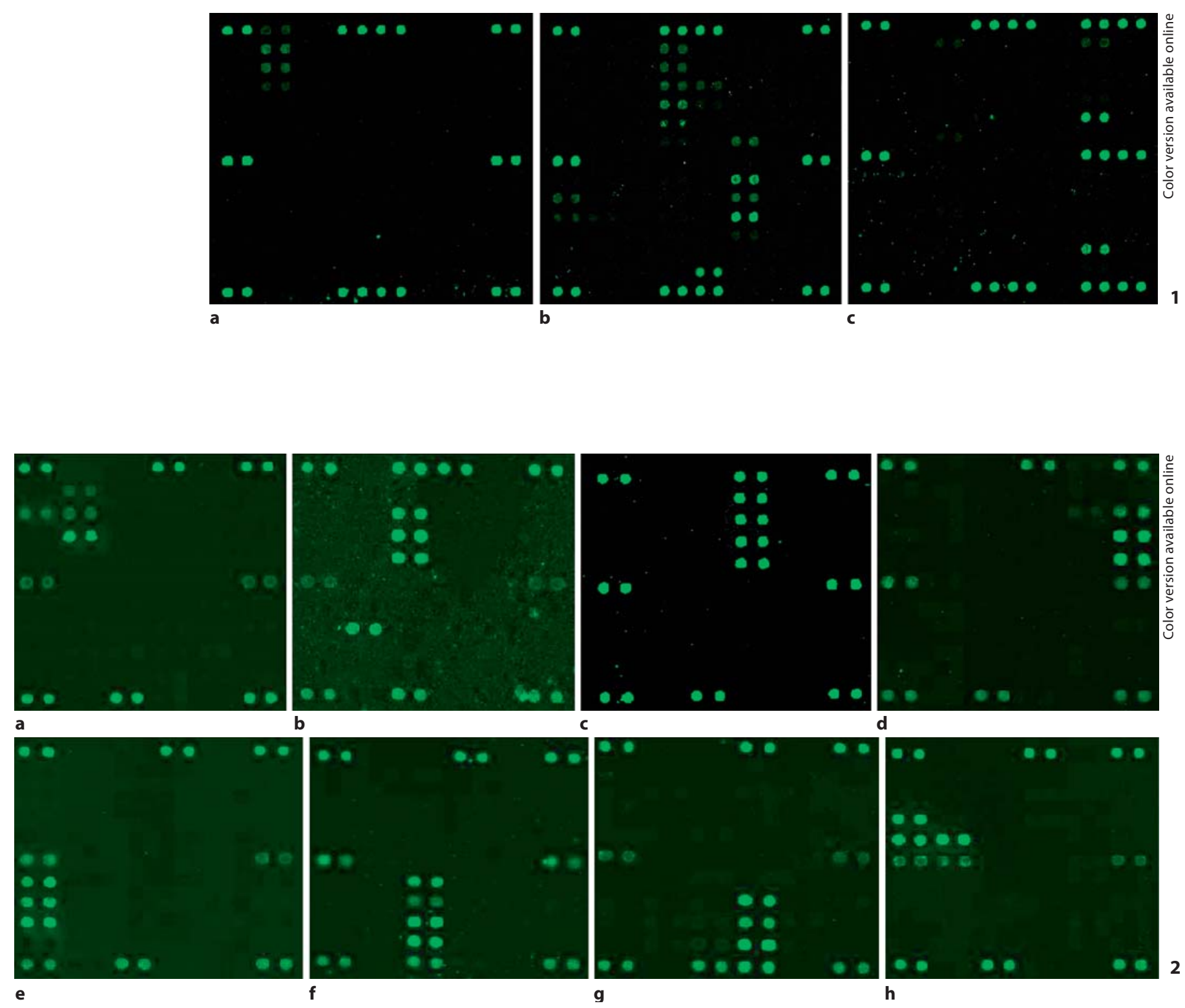

Fig. 1. Detection of gene chip by (a) $\mathrm{BCoV}$ multi-PCR product (table 3, group 1), (b) FIPV multi-PCR product (table 3, group 8), and (c) BCoV multi-PCR product (table 3, group 15).

Fig. 2. Hybridization of coronavirus gene chip with (a) FIPV, (b) $\mathrm{BCoV}$, (c) $\mathrm{HRCoV}$, (d) $\mathrm{TCoV}$, (e) $\mathrm{PRCoV}$, (f) $\mathrm{CCoV}$, (g) FCoV, and (h) TGEV multi-PCR probes.

Fig. 3. Hybridization of coronavirus gene chip with universal primer PCR probes.

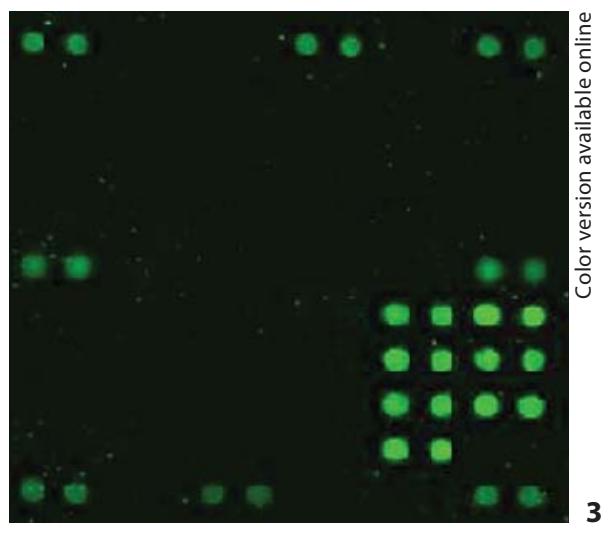


Table 4. Array arrangement of ultimate coronaviruses chip

\begin{tabular}{|c|c|c|c|c|c|c|c|c|c|c|c|}
\hline QC & QC & $\mathrm{BC}$ & $\mathrm{BC}$ & BCoV6 & BCoV6 & QC & QC & TCoV1 & TCoV1 & QC & QC \\
\hline $\mathrm{NC1}$ & $\mathrm{NC} 1$ & FIPV7 & FIPV7 & BCoV7 & BCoV7 & HRCoV3 & HRCoV3 & TCoV2 & TCoV2 & NC2 & $\mathrm{NC} 2$ \\
\hline FIPV2 & FIPV2 & FIPV8 & FIPV8 & BCoV8 & BCoV8 & HRCoV4 & HRCoV4 & TCoV3 & TCoV3 & TCoV6 & TCoV6 \\
\hline TGEV3 & TGEV3 & FIPV9 & FIPV9 & BCoV9 & BCoV9 & HRCoV5 & HRCoV5 & TCoV5 & TCoV5 & TCoV7 & TCoV7 \\
\hline TGEV4 & TGEV4 & TGEV5 & TGEV5 & BCoV10 & BCoV10 & HRCoV12 & HRCoV12 & HRCoV13 & HRCoV13 & TCoV9 & TCoV9 \\
\hline $\mathrm{EC}$ & EC & TGEV6 & TGEV6 & $\mathrm{BC}$ & $\mathrm{BC}$ & HRCoV6 & HRCoV6 & HRCoV15 & HRCoV15 & EC & $\mathrm{EC}$ \\
\hline PRCoV1 & PRCoV1 & $\mathrm{BCoV} 2$ & BCoV2 & CCoV1 & CCoV1 & HRCoV8 & HRCoV8 & UP1 & UP1 & UP10 & UP10 \\
\hline PRCoV2 & PRCoV2 & $\mathrm{BCoV} 3$ & $\mathrm{BCoV} 3$ & CCoV2 & CCoV2 & FCoV6 & FCoV6 & UP3 & UP3 & UP12 & UP12 \\
\hline PRCoV3 & PRCoV3 & BCoV12 & BCoV12 & CCoV5 & CCoV5 & FCoV7 & FCoV7 & UP6 & UP6 & UP14 & UP14 \\
\hline NC3 & NC3 & BCoV13 & BCoV13 & CCoV7 & CCoV7 & FCoV8 & FCoV8 & UP8 & UP8 & NC4 & $\mathrm{NC} 4$ \\
\hline QC & $\mathrm{QC}$ & BCoV14 & BCoV14 & QC & $\mathrm{QC}$ & FCoV9 & FCoV9 & $\mathrm{BC}$ & $\mathrm{BC}$ & $\mathrm{QC}$ & $\mathrm{QC}$ \\
\hline
\end{tabular}
HLA.

$\mathrm{QC}=$ Quality control, $10 \mu \mathrm{M}$ HEX; BC = blank control, 50\% DMSO; NC = negative control, 127 (SARS); EC = PCR product of

Design and Verification of Ultimate Coronavirus Chip

The ultimate specific gene chip was developed with the DNA fragments reamplified from the chosen recombinant plasmids without cross-reaction between different coronaviruses, as shown in table 4. Different multiPCR samples with Cy3-dCTP using specific primer sets were hybridized with the chip and scanned. Intense signals to the positive and control point and no signal to the negative and blank point were shown. Figure $2 \mathrm{a}-\mathrm{h}$ shows obvious hybridization in the ultimate gene chip, with which several coronaviruses could be distinguished easily because of no cross-reaction between different coronaviruses.

\section{Hybridization of Coronavirus Gene Chip with}

\section{Universal Primer PCR Probes}

The mixed cDNA of eight coronaviruses was PCRamplified using a specific universal primer and labeled with Cy3-dCTP, then the PCR product was hybridized with the chip and scanned. The intense signal to the positive and control point and no signal to the negative and blank point, which can determine whether coronavirus exists, are shown in figure 3 . It was suggested that this chip can diagnose at least these eight viruses and possibly more, but this still has to be proven.

\section{Sensitivity and Application of the Coronavirus Chip}

The 10 -fold serial $\mathrm{BCoV}$ cDNA dilution was detected by multi-PCR and the coronavirus chip using the same primer sets of $\mathrm{BCoV} 2, \mathrm{BCoV} 7, \mathrm{BCoV} 9, \mathrm{BCoV} 12, \mathrm{BCoV} 13$ and $\mathrm{BCoV} 14$. The results showed that $10^{-2}$ diluted $\mathrm{CDNA}$ can be detected by multi-PCR and $10^{-5}$ diluted cDNA de- tected by the chip (shown in fig. $4 \mathrm{a}-\mathrm{h}$ ), indicating that the chip may be $1,000 \times$ more sensitive than PCR.

Table 5 shows that the sensitivity of cell culture detection was lower, while the microassay was higher and had comparable sensitivities with PCR. At the same time, the results obtained from field samples with microassay detection showed good concordance with PCR methods.

\section{Discussion}

Microarrays and biosensors have become valuable tools for viral discovery, detection, and genotyping, which have been commonly used in gene expressions, diagnosis of disease, discovery of new genes and drug screening [18-21]. Here we have constructed a coronavirus-specific DNA microarray. The assay was designed to be broadly reactive with the genome of many coronavirus species. It is demonstrated that it can detect eight coronaviruses including the HRCoV-229E which are well-recognized human pathogens.

In order to make a coronavirus microarray useful, it must provide information for a wide range of viral strains and not have a cross-reaction between different coronaviruses. We constructed 104 cDNA clones of eight viruses including different genes by using different (4-15) pairs of primers designed for TCoV, CCoV, FCoV, FIPV, TGEV, PRCoV, BCoV and $\mathrm{HRCoV}$, and a pair of universal primers designed for the RNA polymerase gene of coronavirus. Probes were compared to the database to ensure each probe was unique to the respective virus. The probe fragment was then obtained through plasmid PCR using 
Table 5. Results showing that the sensitivity of cell culture detection was lower, while the microassay was higher and had comparable sensitivities with PCR. At the same time, the results obtained from field samples with microassay detection showed good concordance with PCR methods

\begin{tabular}{|c|c|c|c|c|c|c|c|c|c|c|c|}
\hline $\begin{array}{l}\text { Virus } \\
\text { infected }\end{array}$ & $\begin{array}{l}\text { Animal } \\
\mathrm{n}\end{array}$ & Tissues & RT-PCR & Chip & $\begin{array}{l}\text { Cell } \\
\text { culture }\end{array}$ & $\begin{array}{l}\text { Virus } \\
\text { infected }\end{array}$ & $\begin{array}{l}\text { Animal } \\
\mathrm{n}\end{array}$ & Tissues & RT-PCR & Chip & $\begin{array}{l}\text { Cell } \\
\text { culture }\end{array}$ \\
\hline \multirow[t]{18}{*}{$\mathrm{CCoV}$} & \multirow[t]{6}{*}{ canine (2) } & muscle & - & - & - & \multirow[t]{17}{*}{$\mathrm{FCoV}$} & \multirow[t]{5}{*}{ feline (3) } & muscle & - & - & - \\
\hline & & heart & + & + & - & & & heart & - & - & - \\
\hline & & liver & - & + & - & & & liver & - & + & - \\
\hline & & spleen & - & - & - & & & lung & - & - & - \\
\hline & & lung & - & + & - & & & kidney & - & + & - \\
\hline & & kidney & - & - & - & & \multirow[t]{6}{*}{ feline (3) } & muscle & - & - & - \\
\hline & \multirow[t]{6}{*}{ canine (1) } & muscle & - & - & - & & & heart & - & - & - \\
\hline & & heart & + & + & - & & & liver & - & - & - \\
\hline & & liver & - & - & - & & & spleen & - & - & - \\
\hline & & spleen & - & + & - & & & lung & - & + & - \\
\hline & & lung & - & - & - & & & kidney & - & - & - \\
\hline & & kidney & - & - & - & & \multirow[t]{6}{*}{ feline (2) } & muscle & - & - & - \\
\hline & \multirow[t]{6}{*}{ canine (2) } & muscle & - & - & - & & & heart & - & - & - \\
\hline & & heart & - & & - & & & liver & - & - & - \\
\hline & & liver & - & + & - & & & spleen & - & - & - \\
\hline & & spleen & - & + & - & & & lung & - & - & - \\
\hline & & lung & - & - & - & & & kidney & - & - & - \\
\hline & & kidney & - & - & - & \multirow{12}{*}{ TGEV } & \multirow{6}{*}{ pig (3) } & muscle & - & - & _ \\
\hline \multirow[t]{11}{*}{$\mathrm{BCoV}$} & \multirow[t]{6}{*}{ cattle (2) } & muscle & + & + & - & & & heart & - & + & - \\
\hline & & heart & - & - & - & & & liver & - & + & - \\
\hline & & liver & - & + & - & & & spleen & - & + & - \\
\hline & & spleen & - & - & - & & & lung & - & - & - \\
\hline & & lung & - & - & - & & & kidney & - & - & - \\
\hline & & kidney & - & - & - & & \multirow[t]{6}{*}{ pig (3) } & muscle & - & - & - \\
\hline & \multirow[t]{5}{*}{ cattle (2) } & muscle & - & - & - & & & heart & - & - & - \\
\hline & & heart & - & - & - & & & liver & - & - & - \\
\hline & & liver & - & - & - & & & spleen & - & - & - \\
\hline & & spleen & - & - & - & & & lung & - & - & - \\
\hline & & kidney & - & - & - & & & kidney & - & - & - \\
\hline \multirow[t]{12}{*}{ FIPV } & \multirow[t]{6}{*}{ feline (3) } & muscle & - & + & - & \multirow[t]{12}{*}{$\mathrm{PRCoV}$} & \multirow[t]{6}{*}{ pig (3) } & muscle & - & - & - \\
\hline & & heart & & & & & & heart & - & - & - \\
\hline & & liver & - & + & - & & & liver & - & + & - \\
\hline & & spleen & & & & & & spleen & - & - & - \\
\hline & & lung & + & + & - & & & lung & + & + & - \\
\hline & & kidney & + & + & - & & & kidney & - & - & - \\
\hline & \multirow[t]{6}{*}{ feline (2) } & muscle & + & + & - & & \multirow[t]{6}{*}{ pig (2) } & muscle & - & - & - \\
\hline & & heart & + & + & + & & & heart & - & - & - \\
\hline & & liver & - & + & - & & & liver & - & - & - \\
\hline & & spleen & & & & & & spleen & - & - & - \\
\hline & & lung & + & + & - & & & lung & - & + & - \\
\hline & & kidney & - & + & - & & & kidney & - & - & - \\
\hline
\end{tabular}

Detection of different tissues with RT-PCR, the chip and cell culture. 


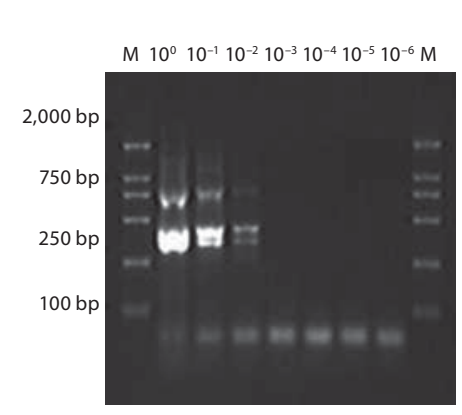

a

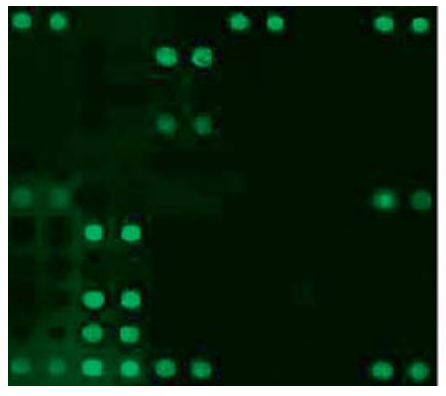

e

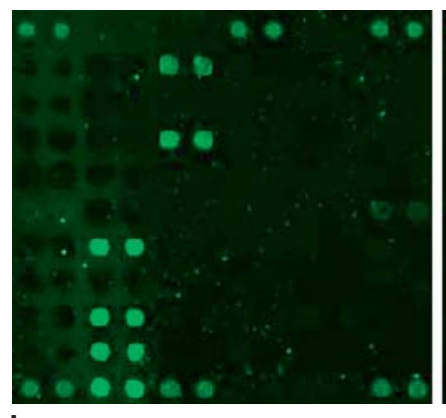

b

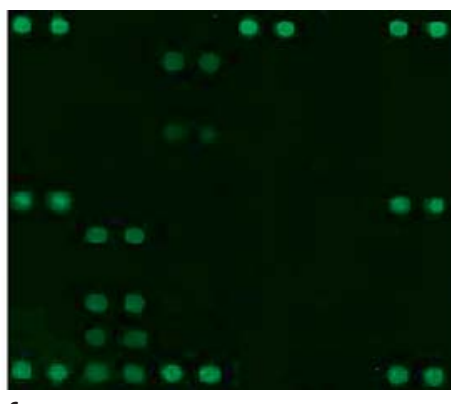

f
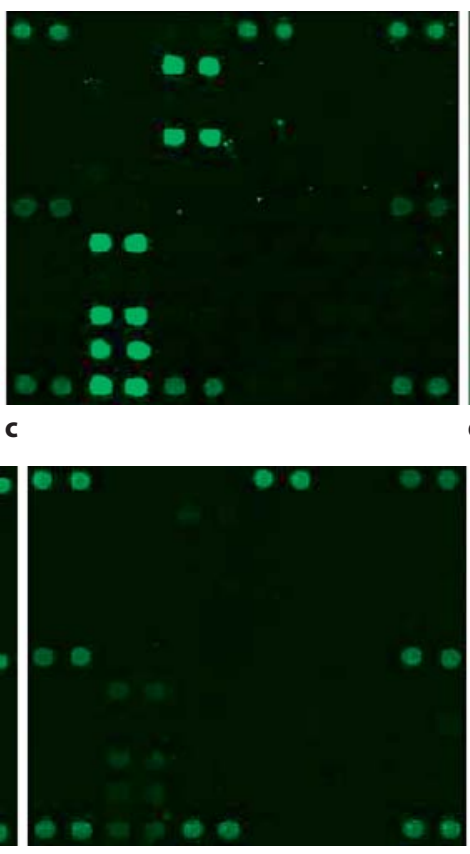

g
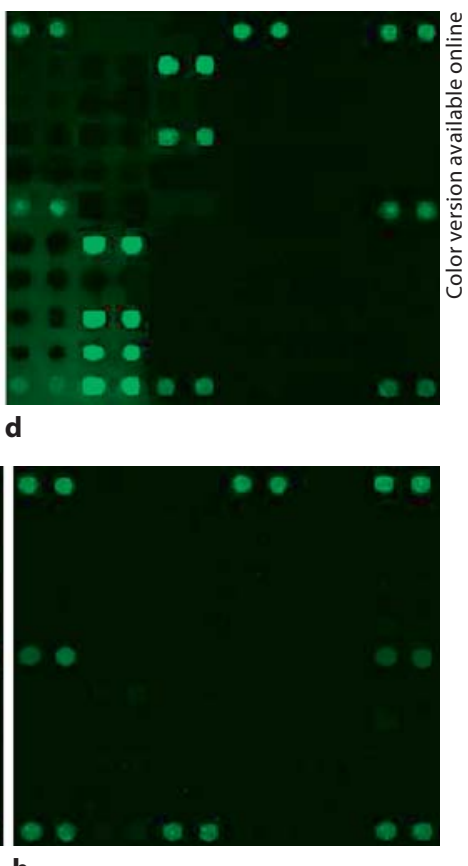

h

Fig. 4. a Detection of $10^{-0}, 10^{-1}, 10^{-2}, 10^{-3}, 10^{-4}, 10^{-5}, 10^{-6} \mathrm{BCoV}$ cDNA by multi-PCR. b-h Hybridization of coronavirus gene chip with (b) $10^{-0} \mathrm{BCoV}$, (c) $10^{-1} \mathrm{BCoV}$, (d) $10^{-2} \mathrm{BCoV}$, (e) $10^{-3} \mathrm{BCoV},(\mathbf{f}) 10^{-4} \mathrm{BCoV},(\mathbf{g}) 10^{-5}$ $\mathrm{BCoV}$, and (h) $10^{-6} \mathrm{BCoV}$ cDNA multi-PCR probes.

the same primers as clone construction, precipitated with ethanol and suspended in $300 \mathrm{ng} / \mu \mathrm{l}$ with $50 \% \mathrm{DMSO}$ for spotting purposes. Viral RNAs were extracted from the cryolysate of cell cultures infected with the examined coronavirus strains, labeled with $\mathrm{Cy} 3$ fluorescent dyes during PCR with different primer pair sets, and hybridized to the primary gene chip. Extensive cross-reactions between $\mathrm{CCoV}, \mathrm{FCoV}$, FIPV, TGEV and PRCoV were found and these clones may exist in a similar site in genes. Through the screening of hybridization, the ultimate specific gene chip was developed with the DNA fragments reamplified from the chosen recombinant plasmids without cross-reaction between different coronaviruses.

In some studies, the sample cDNA was labeled during reverse transcription and then hybridized $[18,22]$. In this study we tried to label the $\mathrm{CDNAs}$ of $\mathrm{BCoV}$ directly with Cy3-dCTP during reverse transcription and subjected them to specific hybridization, however no positive signal appeared. We think the amount of total vRNAs may be so low that the amount of cDNA labeled cannot meet the chip limit required. The amplification of sample cDNAs with PCR is therefore necessary to obtain satis- factory hybridization results. Secondly, the quality and concentration of probes is another important factor related with the signal intensity [23-25]. We obtained the probes by PCR and then purified and concentrated them to make certain that the $\mathrm{OD}_{260}$ was $>0.1$, the $\mathrm{OD}_{260} / \mathrm{OD}_{280}$ was $>1.4$, and the concentration of probes reached 50 $\mu \mathrm{g} / \mathrm{ml}$.

In summary, a new DNA microarray technology is described exhibiting a useful diagnostic method for comprehensive detection of eight coronaviruses - it had a good correlation with PCR and is $1,000 \times$ more sensitive than PCR. It is expected to remain effective with possible mutants and to be of value when other new coronaviruses emerge. Because of the same PCR amplification and further $\mathrm{Cy} 3$ electrochemistry magnification, the chip method has a more than $1,000 \times$ sensitivity than PCR. As we just compare the sensitivity of $\mathrm{BCoV}$ detection using the gene chip and PCR methods with the same primer sets, in future we will compare other coronaviruses and different primer sets with these two methods in order to confirm the high sensitivity of DNA microarray technology. 


\section{References}

1 De Groot RJ, Horzinek MC: Feline infectious peritonitis; in Siddell SG (ed): The Coronaviridae. New York, Plenum Press, 1995, pp 293-315.

2 Xiao X, Feng Y, Chakraborti S: Oligomerization of the SARS-CoV S glycoprotein: dimerization of the $\mathrm{N}$-terminus and trimerization of the ectodomain. Biochem Biophys Res Commun 2004;322:93-99.

- 3 Adach D, Johnson G, Draker R, Ayers M, Mazzulli T, Talbot PJ, Tellier R: Comprehensive detection and identification of human coronaviruses, including the SARS-associated coronavirus with a single RT-PCR assay. J Virol Methods 2004;122:29-36.

4 Pedersen NC, Ward J, Mengeling WL: Antigenic relationship of the feline infectious peritonitis virus to coronaviruses of other species. Arch Virol 1978;58:45-53.

5 Sidde S, Wege H, Meulen V: The biology of coronaviruses. J Gen Virol 1983;64:761776.

-6 Horzinek MC, Lutz H, Pedersen NC: Antigenic relationships among homologous structural polypeptides of porcine, feline and canine coronaviruses. Infect Immun 1982;37:1148-1155.

7 Horsburgh BC, Brierley I, Brown TDK: Analysis of a 9.6-kb sequence from the $3^{\prime}$ end of canine coronavirus genomic RNA. J Gen Virol 1992;73:2849-2862.

-8 Sanchez CM, Jimenez G, Laviada MD, Correa I, Sune C, Builldo M: Antigenic homology among coronaviruses related to transmissible gastroenteritis virus. Virology 1990; 174:410-417.

\9 Wesseling JG, Vennema H, Godeke GJ, Horzinek MC, Rottier PJ: Nucleotide sequence and expression of the spike (S) gene of canine coronavirus with the $S$ protein of feline and porcine coronaviruses. J Gen Virol 1994;75:1789-1794
10 Adeyefa CA, Quayle K, McCauley JW: A rapid method for the analysis of influenza virus genes: application to the reassortment of equine influenza virus genes. Virus Res 1994;32:391-399.

11 Templeton KE, Scheltinga SA, Beersma MFC, Kroes ACM, Claas ECJ: Rapid and sensitive method using multiplex real-time PCR for diagnosis of infections by influenza $A$ and influenza $B$ viruses, respiratory syncytial virus, and parainfluenza viruses 1,2 3, and 4. J Clin Microbiol 2004;42:15641569.

12 Wang Q, Zhang J, Li J: Detection of canine coronavirus in dog fecal samples by RT-PCR. Anim Husb Vet Med 2005;37:1-4.

13 Li H, McCormac MA, Estes RW, Sefers SE, Dare RK, Chappell JD, Erdman DD, Wright PF, Tang YW: Simultaneous detection and high-throughput identification of a panel of RNA viruses causing respiratory tract infections. J Clin Microbiol 2007;45:2105-2109.

14 Liu Q, Bai Y, Ge Q, Zhou S, Wen T, Lu Z: Microarray-in-a-tube for detection of multiple viruses. Clin Chem 2007;53:2188-2194.

$\checkmark 15$ Kleber de Souza Luna L, Heiser V, Regamey N, Panning M, Drexler JF, Mulangu S, Poon L, Baumgarte S, Haijema BJ, Kaiser L, Drosten C: Genetic detection of coronaviruses and differentiation at the prototype strain level by reverse transcription-PCR and nonfluorescent low-density microarray. J Clin Microbiol 2007;45:1049-1052.

16 Long WH, Xiao HS, Gu XM, Zhang QH, Yang HJ, Zhao GP, Liu JH: A universal microarray for detection of SARS coronavirus. J Virol Methods 2004;121:57-63.
17 Sambrook J, Fritsch EF, Maniatis T: Molecular Cloning: a Laboratory Manual, ed 2. Cold Spring Harbor, Cold Spring Harbor Laboratory Press, 1989.

18 Schena M, Shalon D, Davis RW, Brown PO: Quantitative monitoring of gene expression patterns with a complementary DNA microarray. Science 1995;270:467-470.

19 Favis R, Day JP, Gerry NP, Phelan C, Narod S, Barany F: Universal DNA array detection of small insertions and deletions in BRCA1 and BRCA2. Nat Biotechnol 2000;18:561564 .

20 Vernet G: DNA chip technology and infectious diseases. Virus Res 2002;82:65-71.

-21 Kawaguchi K, Kaneko S, Honda M, Kawai HF, Shirota Y, Kobayashi K: Detection of hepatitis B virus DNA in sera from patients with chronic hepatitis $B$ virus infection by DNA microarray method. J Clin Microbiol 2003;41:1701-1704.

22 Townsend MB, Dawson ED, Mehlmann M, Smagala JA, Dankbar DM, Moore CL, Smith CB, Cox NJ, Kuchta RD, Rowlen KL: Experimental evaluation of the FluChip diagnostic microarray for influenza virus surveillance. J Clin Microbiol 2006;44:2863-2871.

23 Marshall A, Hodgson J: DNA chips: an array of possibilities. Nat Biotechnol 1998;16:731736.

24 Tran PH, Peiffer DA, Shin Y: Microarray optimization: increasing spot accuracy and automated identification of true microarray signals. Nucleic Acids Res 2002;30:54-61.

25 Yuen T, Wurmbach E, Pfeffer RL, Ebersole BJ, Sealfon SC: Accuracy and calibration of commercial oligonucleotide and custom cDNA microarrays. Nucleic Acids Res 2002; 30:e48. 